\title{
Transformação box-cox em experimentos com pimentão em ambiente protegido
}

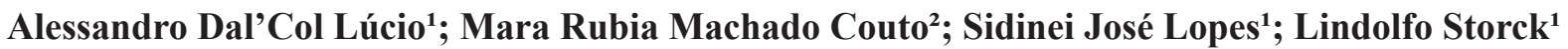 \\ ${ }^{1}$ UFSM-CCR, Depto. Fitotecnia, 97105-900 Santa Maria-RS; 2UFSM-CCR, Depto. Estatística; adlucio@smail.ufsm.br (autor correspon- \\ dente); sjlopes@smail.ufsm.br; lindolfo@smail.ufsm.br; mararubiacouto@hotmail.com
}

\begin{abstract}
RESUMO
Em olericultura, o interesse é a produção de frutos comercializáveis. Em experimentos nessa área agronômica comumente são avaliadas variáveis tais como a fitomassa e o número de frutos. No entanto, essas variáveis apresentam uma característica particular que está relacionada com sua ausência no momento da colheita. Este atributo é representado por valores zero, gerando certa variabilidade e afetando a análise de variância dos dados. Assim, teve-se por objetivo definir uma transformação adequada para as variáveis observadas em colheitas por planta de experimentos com a cultura do pimentão, realizados em ambiente protegido, visando estabilizar a variabilidade gerada pela presença de valores zero nas múltiplas colheitas dos frutos. Os experimentos foram realizados nas estações sazonais verão/outono e inverno/primavera, utilizando a família de transformações de Box-Cox, com uma adaptação apresentada em Yamamura (1999). Apesar da redução na variabilidade e a normalidade dos resíduos, observadas em todos os experimentos, o uso do método não foi eficiente para tornar as variâncias homocedásticas. Concluiu-se que para dados de colheitas por planta de experimentos com pimentão, avaliando a fitomassa e o número de frutos, a transformação indicada é a inversa da raiz quarta.
\end{abstract}

Palavras-chave: Capsicum annuum, transformação de dados, análise de variância, coeficiente de variação, variâncias heterocedásticas.

\begin{abstract}
Box-cox transformation in experiments with green pepper in protected environments

The major purpose of vegetable crops production systems is the profitable and economically viable yield of marketable products. Numerous field experiments with vegetable crops are carried out to determine biomass production of marketable and nonmarketable variables. However, these variables possess the particular characteristic related to their presence or absence at harvest time. Absent variables, represented by zero values, generate variability and affect variance analyses. This research deals with the search of applicable transformations for variables studied in green pepper field experiments carried out in protected environments attempting to stabilize the variability resulting from the presence of zero values in multiple harvests. The analysis was based on experiments with the green pepper crop carried out in summer-fall or winter-spring season, using an adaptation of the Box-Cox transformation family found in Yamamura (1999). Despite the reduction in variability and normal errors, observed in all experiments, the use of the method was not efficient to make the variances homocedastic. For experiments with green pepper, assessing the phytomass and number of fruits, the inverse fourth root transformation is indicated.
\end{abstract}

Keywords: Capsicum annuum, data transformation, analysis of variance, coefficient of variation, heterocedastic variances.

\section{(Recebido para publicação em 17 de agosto de 2009; aceito em 11 de janeiro de 2011) (Received on August 17, 2009; accepted on January 11, 2011)}

$\mathrm{O}$ pimentão (Capsicum annuum) é uma das culturas mais indicadas para ser utilizada em ambiente protegido, situando-se entre as cinco culturas de maior área cultivada no Brasil e em outros países do mundo. Essa cultura é muito utilizada em ambiente protegido, em função da grande produtividade que pode ser alcançada nestas condições, podendo chegar a $150 \mathrm{t} / \mathrm{ha}$, aproximadamente duas vezes o que é obtido normalmente em cultivo em campo, confirmando resultados apresentados por Andriolo et al. (1991), Cunha et al. (2002) e Cunha \& Escobedo (2003).

Em cultivos olerícolas sob ambiente protegido, a maioria dos tratos culturais é realizada de forma individualizada (por planta) e o mais uniforme possível. Porém, alguns autores relatam a existência de variabilidade da produção de frutos entre as linhas de cultivo, o que pode prejudicar a condução de experimentos de pesquisa nessas condições. Souza et al. (2002) e Mello et al. (2004) relataram grande variabilidade para crescimento dos frutos de abobrinha italiana no decorrer do ciclo produtivo. Da mesma forma, Lúcio et al. (2003) observaram que em $100 \%$ das colheitas de pimentão havia heterogeneidade das variâncias entre as plantas. Já Lorentz et al. (2005), trabalhando com pepineiro, verificaram que $46 \%$ das colheitas mostraram-se com variâncias heterogêneas entre plantas, observando também grandes oscilações das médias da produção por colheita. Também, Lopes et al. (1998) relataram mudanças na concentração da produção de tomate no decorrer das colheitas e constataram diferenças de produção entre as linhas de cultivo.

A variabilidade da produção entre plantas sob cultivo protegido pode acarretar em heterogeneidade de variâncias que, para realização da ANOVA, deverão ser rejeitadas e, assim, assegurar o nível de significância dos testes $\mathrm{F}$ e os de comparação múltipla de médias. Em análises de dados experimentais com variâncias heterogêneas, o nível de significância passa a ficar acima do especificado (Conagin et al., 1993). Assim, se faz necessário o estudo da resposta 
da média e da variância da produção em ambiente protegido, ao longo de diferentes colheitas, bem como a interferência direta dos diferentes manejos culturais nessas estatísticas, pois são fatores que podem alterar as conclusões de trabalhos científicos. No entanto, a ocorrência de valores zero em experimentos com culturas olerícolas com múltiplas colheitas é frequente, pois nem sempre existem frutos a serem colhidos ou, nem sempre, estes possuem tamanho adequado para sua colheita e comercialização (Souza et al., 2002; Mello et al. 2004; Lorentz et al., 2005; Carpes et al., 2008; Lúcio et al., 2008). Nestes casos, o uso de transformações de variáveis se faz necessário, para que se possa atingir a homocedasticidade na análise de variância. Mesmo assim, a eficácia das transformações fica prejudicada, especialmente aquelas que empregam a função logarítmica, inclusive a família de transformações Box-Cox (Box \& Cox, 1964). Estudos que descrevem a variabilidade de populações utilizam frequentemente a transformação logarítmica para alcançar a homocedasticidade ou a estabilidade da variância para testar os efeitos na ANOVA.

Ao se dispor de um conjunto de dados com valores iguais a zero é comum o acréscimo de uma constante (c) a cada um dos dados (Banzatto \& Kronka, 1989; Storck et al., 2000). A determinação do valor constante $c$ que deve ser adicionado à variável original $(\mathrm{Y})$ tem sido bastante discutida na literatura mas, usualmente, o valor da constante que é somado a todos os dados é igual à unidade. Conforme Yamamura (1999), não há base concreta quanto às razões pelas quais a unidade é adicionada ao invés de outra constante, tais como 0,5 ou dois. Outros princípios alternativos foram propostos por Hill (1963), Griffiths (1980) e Berry (1987) para determinar o parâmetro c. Os procedimentos propostos por esses autores são aplicáveis em um determinado conjunto de pressupostos, os quais requerem cálculos complexos. Devido a este fato, um princípio prático foi proposto por Yamamura (1999) sugerindo como escolha razoável para o parâmetro $c$ o valor 0,5 , se a forma da distribuição não for conhecida. $\mathrm{O}$ autor defende o uso de $c$ igual a 0,5 pelo motivo que, se uma distribuição discreta definida em $0,1,2$, ..., pode ser aproximadamente descrita por uma distribuição contínua definida em $0, \infty$ quando usa-se $c=0,5$. Trabalhando com abobrinha italiana, Couto et al. (2009) adotaram esse procedimento obtendo como transformação adequada a raiz quarta tanto para o número quanto para a fitomassa dos frutos colhidos, apresentada como uma alternativa para estabilizar a variância ou, pelo menos, reduzir a variabilidade existente.

Este trabalho teve por objetivo definir uma transformação adequada para as variáveis observadas em experimentos com a cultura do pimentão, realizados em ambiente protegido, visando estabilizar a variabilidade gerada pela ausência de valores nas múltiplas colheitas dos frutos por planta.

\section{MATERIAL E MÉTODOS}

Foram realizados dois experimentos com pimentão, cultivar Vidi, nas épocas de cultivo verão/outono (época 1) e inverno/primavera (época 2), em área experimental do Departamento de Fitotecnia da Universidade Federal de Santa Maria (294' $\mathrm{S}, 5^{\circ} 43^{\prime} \mathrm{W}$, altitude 95 $\mathrm{m})$, tendo clima classificado como Cfa (subtropical úmido sem estação seca definida e com verões quentes) e solo classificado como Argissolo Vermelho Distrófico Arênico. Em cada época, o cultivo foi realizado em estufa plástica com dimensões de $24 \mathrm{~m}$ de comprimento por $10 \mathrm{~m}$ de largura, com 3,5 m de altura na parte central, utilizando irrigação por gotejamento, camalhões com mulching preto de PEDB de 35 micras de espessura com $0,1 \mathrm{~m}$ de altura e $0,4 \mathrm{~m}$ de largura, dez linhas de cultivo, 70 plantas cada, sendo o espaçamento de $0,6 \mathrm{~m}$ entre as filas e 0,3 m entre as plantas. As aplicações de fungicidas, inseticidas e as desfolhas das plantas foram realizadas sempre que necessário nas duas épocas, conforme recomendação da cultura (Filgueira, 2000).

As plantas foram identificadas com o número de ordem da linha e da planta dentro de cada linha. A definição do momento da colheita baseou-se no tamanho dos frutos, onde estes foram diariamente verificados e colhidos quan- do apresentaram a coloração de verde para verde azulado. Os frutos colhidos foram colocados em sacos de papel e identificados, para posterior determinação, em cada colheita, do número de frutos colhidos por planta e da fitomassa fresca, empregando-se balança digital com precisão de um grama.

Utilizou-se a metodologia Box-Cox (Box \& Cox, 1964) para o conjunto de funções potências, a fim de encontrar a transformação para estabilizar ou reduzir a variabilidade existente e normalizar os resíduos. Como para valores nulos, a família de transformações de BoxCox fica restrita, utilizou-se a variável somada a uma constante. Adotou-se o procedimento proposto por Yamamura (1999), com o parâmetro $c=0,5$, gerando assim as expressões:

$$
\mathrm{f}(\mathrm{y})=\frac{(\mathrm{y}+0,5)^{\lambda}-1}{\lambda}, \lambda \neq 0 \quad, \mathrm{e}
$$

$$
f(y)=\ln (y+0,5), \lambda=0
$$

que é a família de transformações BoxCox, acrescida do parâmetro $c=0,5$.

Para a escolha da melhor potência $\lambda$, foram considerados valores no intervalo $[-2,2]$, conforme descrevem Draper \& Smith (1981), sendo substituídos nas expressões:

$$
\begin{aligned}
& Z^{(\lambda)}=\frac{\mathrm{Y}^{\lambda}-1}{\lambda G^{\lambda-1}}, \text { para } \lambda \neq 0 \text { ou } \\
& Z^{(\lambda)}=G \ln Y \text {, para } \lambda=0 \\
& \text { em a quantidade (constante) }
\end{aligned}
$$
$G=\left(Y_{1} Y_{2} Y_{3} \ldots Y_{N}\right)^{\frac{1}{N}}$ é um estimador da média geométrica de $Y_{i}$ ou seja, do conjunto de dados e o jacobiano $J(\lambda, z)=1$, gerando valores transfor$\operatorname{mados}\left(Z^{(\lambda)}\right)$ para cada variável.

Foi realizada a análise de variância para cada variável transformada e para cada valor de $\lambda$, adotando o delineamento em blocos ao acaso em faixas, com o modelo matemático descrito por: $\mathrm{Y}_{\mathrm{ijk}}=$ $\mathrm{m}+\mathrm{b}_{\mathrm{j}}+\mathrm{A}_{\mathrm{i}}+\mathrm{ea}_{\mathrm{ij}}+\mathrm{D}_{\mathrm{k}}+\mathrm{ed}_{\mathrm{jk}}+\mathrm{AD}_{\mathrm{ik}}+\mathrm{e}_{\mathrm{ijk}}$, onde $\mathrm{Y}_{\mathrm{ijk}}=$ valor da variável observada, $\mathrm{b}_{\mathrm{j}}=$ efeito aleatório do bloco $\mathrm{j}$ (linhas de cultivo), $\mathrm{A}_{\mathrm{i}}=$ efeito fixo do fator A (posi- 
ção da planta na linha de cultivo) em faixas horizontais, $\mathrm{ea}_{\mathrm{ij}}=$ efeito aleatório da interação entre o bloco j e o fator A em faixas (erro A), $D_{k}=$ efeito fixo do fator $\mathrm{D}$ (colheitas realizadas) em faixas verticais, $e_{\mathrm{jk}}=$ efeito aleatório da interação entre o bloco $\mathrm{j}$ e o fator $\mathrm{D}$ em faixas (erro D), $\mathrm{AD}_{\mathrm{ik}}=$ interação entre os i níveis do fator $\mathrm{A}$ e os $\mathrm{k}$ níveis do fator $\mathrm{D}$ e $\mathrm{e}_{\mathrm{ijk}}=$ erro experimental. Os tratamentos foram simulados com a combinação das 70 plantas em cada linha de cultivo com as 10 colheitas realizadas. Para determinar o estimador de máxima verossimilhança $\left(L_{\max }\right)$, substituiu-se a soma de quadrados residual $\left(S S E_{(\lambda)}\right)$, encontrada para cada valor de $\lambda$ usado, na expressão:

$L_{\max (\lambda)}=-\frac{n}{2} \ln \left(\frac{\hat{\sigma}_{\lambda}^{2}(z)}{n}\right)=-\frac{n}{2} \ln \left(\frac{\operatorname{SSE}(\lambda)}{n}\right)$

sendo que o melhor $\lambda$ foi escolhido de maneira que maximizasse $L_{\max }$.

Foram obtidas as medidas descritivas e a análise de variância para as variáveis, antes e após o uso de transfor- mação, aplicando o teste de Bartlett para verificar se a transformação foi eficiente para satisfazer a homocedasticidade das variâncias residuais, após eliminação do efeito de bloco e, finalmente, foi realizada a análise dos resíduos antes e após a transformação. Para a análise dos dados foi adotado $5 \%$ de probabilidade de erro.

\section{RESULTADOS E DISCUSSÃO}

A frequência de valores zero, obtidos nas colheitas para as variáveis número e fitomassa de frutos de pimentão, variou em torno de $40 \%$ no inverno/primavera (I/P) e 35\% no verão/outono (V/O). Esse comportamento indica que a maior variabilidade para as variáveis estudadas foi obtida na estação de cultivo I/P, concordando com os resultados obtidos por Lúcio et al. (2003) e Lorentz et al. (2005). Essa heterogeneidade das variâncias no I/P deve-se, principal- mente, à grande variabilidade existente entre o crescimento das plantas e dos frutos, causada pelas maiores variações nas condições ambientais no ambiente protegido, tais como temperatura do ar, radiação solar global, nebulosidade e umidade relativa do ar, que afetam o crescimento dos frutos e a definição do ponto de colheita dos mesmos. Na cultura do pimentão, os frutos surgem em dias diferentes de uma planta para outra, de acordo com a formação das novas brotações que despontam no decorrer do desenvolvimento das plantas, provocando um crescimento desuniforme entre os frutos (Lorentz et al., 2005). Assim, a heterogeneidade das variâncias é devida, principalmente, ao amadurecimento precoce ou tardio de alguns frutos, causados por alterações nas condições fisiológicas ou condições ambientais adversas, mais proeminentes na estação I/P.

O valor da potencia $\lambda$ que minimizou a raiz do quadrado médio do erro (RQME) foi de $-0,25$ (Tabela 1 ),

Tabela 1. Função de máxima verossimilhança $\left(\mathrm{L}_{\max }\right)$ e raiz do quadrado médio do erro (RQME) obtidos pelo procedimento Box-Cox na escolha da potência $\lambda$, para as variáveis número de frutos (NF) e fitomassa fresca de frutos (FFF), em gramas, de pimentão cultivado em ambiente protegido nas estações sazonais verão/outono $(\mathrm{V} / \mathrm{O})$ e inverno/primavera $(\mathrm{I} / \mathrm{P})$ (function of maxim verisimilitude $\left(\mathrm{L}_{\text {max }}\right)$ and root of the mean square of the error (RQME) obtained by the procedure Box-Cox in the choice of the potency $\lambda$, for the variables number of fruits (NF) and fresh biomass of fruits (FFF), in grams, of green pepper cultivated in protected environments carried out in summer-fall (V/O) or winter-spring (I/P) season). Santa Maria, UFSM, 2009.

\begin{tabular}{|c|c|c|c|c|c|c|c|c|}
\hline \multirow{3}{*}{$\lambda$} & \multicolumn{4}{|c|}{$\mathrm{V} / \mathrm{O}$} & \multicolumn{4}{|c|}{$\mathbf{I} / \mathbf{P}$} \\
\hline & \multicolumn{2}{|c|}{ NF } & \multicolumn{2}{|c|}{ FFF } & \multicolumn{2}{|c|}{ NF } & \multicolumn{2}{|c|}{ FFF } \\
\hline & $\mathbf{L}_{\max }$ & RQME & $\mathbf{L}_{\max }$ & RQME & $\mathbf{L}_{\max }$ & RQME & $\mathbf{L}_{\max }$ & RQME \\
\hline$-2,00$ & $-54278,4$ & 54,0 & $-48140,6$ & 34,4 & $-42780,2$ & 50,9 & $-38005,6$ & 32,8 \\
\hline$-1,75$ & $-48503,8$ & 35,3 & $-43174,9$ & 23,9 & $-38252,0$ & 33,6 & $-33999,5$ & 22,7 \\
\hline$-1,50$ & $-42995,9$ & 23,6 & $-38496,2$ & 16,9 & $-33932,7$ & 22,6 & $-30223,5$ & 16,1 \\
\hline$-1,25$ & $-37844,3$ & 16,1 & $-34177,8$ & 12,3 & $-29890,9$ & 15,6 & $-26743,7$ & 11,7 \\
\hline$-1,00$ & $-33184,6$ & 11,5 & $-30322,8$ & 9,3 & $-26231,4$ & 11,1 & $-23653,5$ & 8,8 \\
\hline$-0,75$ & $-29223,8$ & 8,6 & $-27082,4$ & 7,3 & $-23114,9$ & 8,4 & $-21087,4$ & 6,9 \\
\hline$-0,50$ & $-26260,8$ & 6,9 & $-24678,9$ & 6,1 & $-20777,9$ & 6,7 & $-19235,5$ & 5,9 \\
\hline$-0,25$ & $-24657,3$ & 6,1 & $-23415,5$ & 5,6 & $-19513,2$ & 6,0 & $-18339,6$ & 5,4 \\
\hline 0,00 & $-24698,3$ & 6,1 & $-23614,5$ & 5,7 & $-19561,8$ & 6,0 & $-18633,0$ & 5,5 \\
\hline 0,25 & $-26413,4$ & 7,0 & $-25454,8$ & 6,5 & $-20963,1$ & 6,9 & $-20221,2$ & 6,4 \\
\hline 0,50 & $-29570,2$ & 8,8 & $-28842,8$ & 8,3 & $-23537,6$ & 8,7 & $-23010,4$ & 8,3 \\
\hline 0,75 & $-33842,8$ & 12,0 & $-33485,9$ & 11,7 & $-27022,2$ & 12,0 & $-26775,3$ & 11,7 \\
\hline 1,00 & $-38948,5$ & 17,5 & $-39065,9$ & 17,6 & $-31185,6$ & 17,5 & $-31277,6$ & 17,7 \\
\hline 1,25 & $-44680,8$ & 26,7 & $-45333,1$ & 28,0 & $-35858,3$ & 26,9 & $-36327,7$ & 28,1 \\
\hline 1,50 & $-50894,9$ & 42,1 & $-52113,2$ & 46,0 & $-40921,1$ & 42,9 & $-41789,8$ & 46,4 \\
\hline 1,75 & $-57487,3$ & 68,3 & $-59287,2$ & 78,0 & $-46289,4$ & 70,2 & $-47569,2$ & 79,0 \\
\hline 2,00 & $-64382,4$ & 113,4 & $-66771,4$ & 135,1 & $-51901,7$ & 117,6 & $-53598,3$ & 137,4 \\
\hline
\end{tabular}


Tabela 2. Média ( $\overline{\mathrm{X}})$, quadrado médio do erro (QMe) e coeficiente de variação (CV\%) para as variáveis original e transformadas mais a constante para o número de frutos (NF) e fitomassa fresca de frutos (FFF), em gramas, de pimentão cultivado em ambiente protegido nas estações sazonais verão/outono $(\mathrm{V} / \mathrm{O})$ e inverno/primavera $(\mathrm{I} / \mathrm{P})($ means $(\overline{\mathrm{X}})$, mean square of the error $(\mathrm{QMe})$ and variation coefficient $(\mathrm{CV} \%)$ for original and transformed variables and the constant for the number of fruits (NF) and fresh biomass of fruits (FFF), in grams, of green pepper cultivated in protected environments during summer-fall (V/O) or winter-spring (I/P) seasons). Santa Maria, UFSM. 2009.

\begin{tabular}{|c|c|c|c|c|c|c|}
\hline \multirow{2}{*}{ Variáveis } & \multicolumn{3}{|c|}{$\mathbf{V} / \mathbf{O}$} & \multicolumn{3}{|c|}{$\mathbf{I} / \mathbf{P}$} \\
\hline & $\bar{X}$ & QMe & CV $(\%)$ & $\bar{X}$ & QMe & CV $(\%)$ \\
\hline FFF & 0,22 & 0,04 & 90,91 & 0,31 & 0,08 & 91,24 \\
\hline$(\mathrm{FFF}+0,5)^{-0,25}$ & 1,10 & 0,03 & 15,74 & 1,08 & 0,07 & 7,75 \\
\hline NF & 1,31 & 1,27 & 86,03 & 1,43 & 1,48 & 85,07 \\
\hline$(\mathrm{NF}+0,5)^{-0,25}$ & 0,94 & 0,03 & 18,43 & 0,94 & 0,03 & 18,42 \\
\hline
\end{tabular}

indicando que a melhor transformação para os dados é o inverso da raiz quarta $\left(\mathrm{y}^{-0,25}\right)$. Nesse caso, de acordo com Box \& Cox (1964), a expressão:

$Z^{(\lambda)}=\frac{Y^{\lambda}-1}{\lambda}$, para $\lambda \neq 0 ;$ $\mathrm{Z}^{(\lambda)}=\ln \mathrm{Y}$, para $\lambda=0 \operatorname{com} \mathrm{Y}>0$

é equivalente a $Z^{(\lambda)}=Y^{\lambda}$, para $\lambda \neq 0$ e $Z^{(\lambda)}=\ln \mathrm{Y}$, para $\lambda=0$ quando a análise de variância é invariante com transformações lineares. A relação direta entre as variáveis fitomassa e o número de frutos colhidos pode ter sido um dos motivos para o qual se tem o mesmo valor $\lambda$ para essas duas variáveis.

As medidas descritivas para o peso e o número de frutos sem e com o acréscimo da constante, antes e após a transformação, indicam que ao se aplicar a transformação na variável acrescida da constante houve uma redução no coeficiente de variação, de $78 \%$ e de $79 \%$ na variável número de frutos no $\mathrm{I} / \mathrm{P}$ e $\mathrm{V} / \mathrm{O}$, respectivamente, e de $92 \%$ e $83 \%$ para fitomassa de frutos no I/P e $\mathrm{V} / \mathrm{O}$, em comparação à variável original mais 0,5 , indicando uma redução da variabilidade dos dados (Tabela 2). Essa redução é interessante, pois a diminuição no desvio padrão foi muito mais evidente que o aumento na estimativa da média para o número de frutos, o que se espera de uma transformação de dados para homogeneizar variâncias.

Pelos resultados obtidos nas análises de variância para o número e a fitomassa fresca de frutos para as duas épocas de cultivo, houve também uma redução no quadrado médio do erro (QME) após o uso da transformação, da ordem de $25 \%$ e de $12 \%$ na estação $\mathrm{V} / \mathrm{O}$, respectivamente, para número e fitomassa fresca de frutos e de 98\% na estação I/P para as duas variáveis estudadas (Tabela 2). Este resultado é importante, pois quanto maior o erro, menor a probabilidade de se encontrarem diferenças significativas entre médias de tratamentos. Portanto, a transformação inversa da raiz quarta para o número e o peso de frutos de pimentão permitiu uma redução na variabilidade, principalmente na estação que apresentou maior frequência de valores zero para as variáveis em estudo. Esse resultado também foi obtido por Couto et al. (2009) estudando a cultura da abobrinha italiana, com a transformação raiz quarta, evidenciando a eficácia da metodologia de transformação de dados na homogeneidades das variâncias.

A transformação inversa da raiz quarta não foi eficiente para adequar os dados à pressuposição da homogede Bartlett rejeitou-se a hipótese de homocedasticidade para o número e a fitomassa de frutos nas duas estações de cultivo, apesar da redução no valor do $\chi^{2}$ calculado para as duas variáveis avaliadas. Sabe-se que muitas circunstâncias podem interferir na violação da pressuposição de homogeneidade de variância. Nesse trabalho, as variáveis avaliadas apresentaram melhoras significativas ao atendimento à normalidade neidade das variâncias, pois pelo teste dos erros com os dados transformados para ambas as variáveis (Figura 1). Mesmo rejeitando-se a hipótese de homocedasticidade destes, o nível de probabilidade apresentado no teste, após a transformação foi de $p=0,0615$, muito próximo do adotado no estudo $(p<0,05)$. A transformação inversa da raiz quarta também proporcionou a menor relação entre $\mathrm{RQME} \mathrm{e} \mathrm{L}_{\max }$, indicando a melhor condição possível para transformação dos dados e homogeneidade dos erros. Mesmo não sendo confirmada a homogeneidade das variâncias, a transformação inversa da raiz quarta é uma possibilidade que permite estabilizar a variância ou, pelo menos reduzi-la, auxiliando nas análises estatísticas e interpretações dos resultados deste tipo de experimento com múltiplas colheitas, sem comprometer a precisão experimental.

Para o número e a fitomassa fresca de frutos, avaliados em colheitas por planta, em experimentos com a cultura do pimentão em ambiente protegido, a transformação indicada é a inversa da raiz quarta.

\section{AGRADECIMENTOS}

À FAPERGS, ao CNPq e ao Programa Especial de Incentivo às Publicações Internacionais/PRPGP/UFSM pelo auxílio financeiro para a realização do trabalho e pela concessão de bolsas de produtividade em pesquisa aos autores Alessandro Dal'Col Lúcio, Sidinei José Lopes e Lindolfo Storck.

\section{REFERÊNCIAS}

ANDRIOLO JL; BURIOL GA; STRECK NA; FIORIN J. 1991. Influência da proteção ambiental com estufa de polietileno transparente sobre o crescimento e desenvolvimento do pimentão. Horticultura Brasileira 21: 191204.

BANZATTO DA; KRONKA SN. 1989. Experimentação agrícola. 1ed. Jaboticabal: FUNEP, 247p.

BERRY DA. 1987. Logarithmic transformations in ANOVA. Biometrics 43: 439-456.

BOX GEP; COX DR. 1964. An analysis of transformations. Journal of the Royal Society, 26: 211-252.

CARPES RH; LÚCIO AD; STORCK L; LOPES SJ; ZANARDO B; PALUDO AL. 2008. Ausência de frutos colhidos e suas interferências nas estimativas da fitomassa 


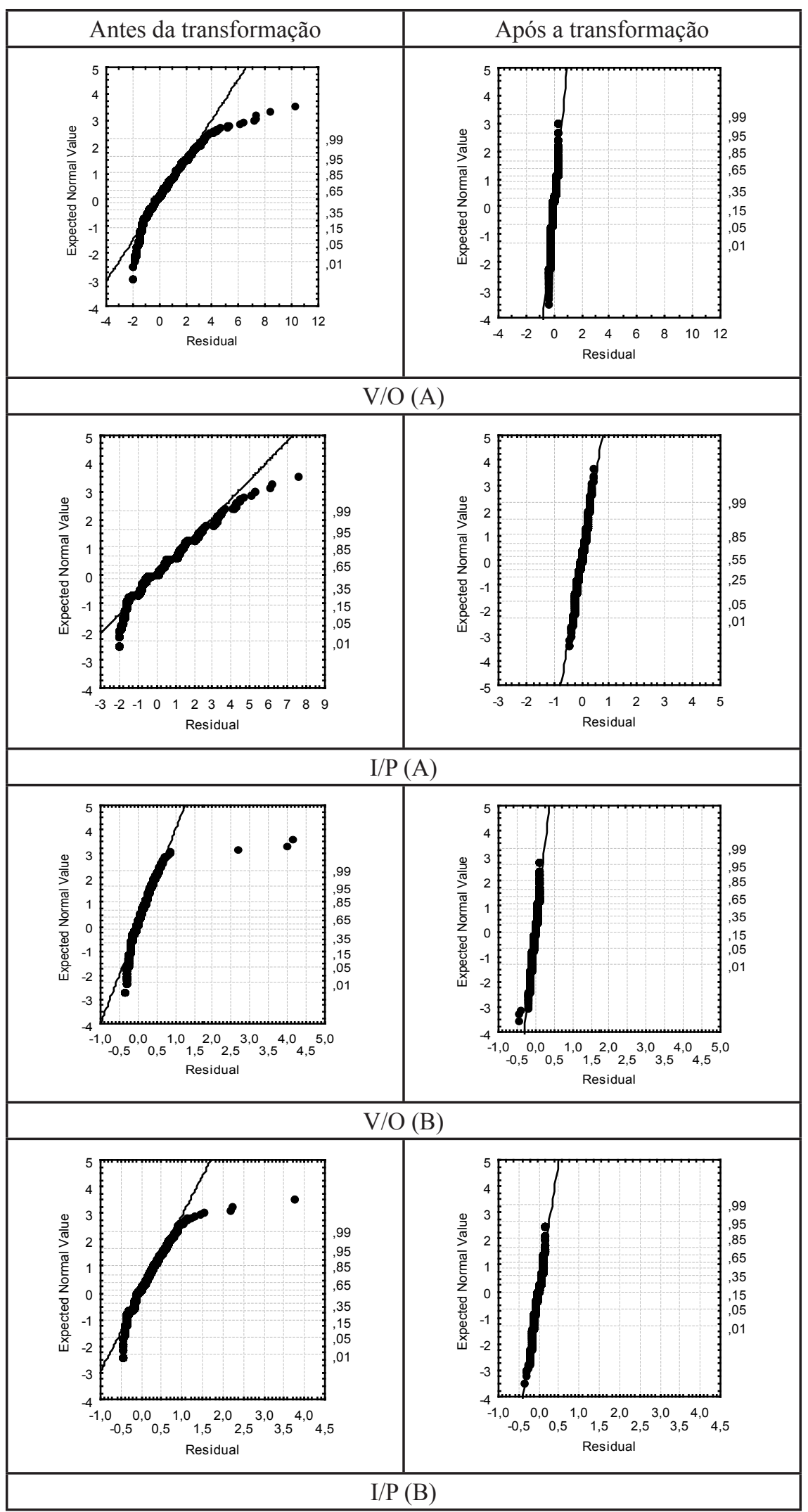

Figura 1. Resíduos (Residual) em função do valor normal (Expected normal value) para número de frutos $(\mathrm{A})$ e fitomassa fresca de frutos $(\mathrm{B})$ antes e após a transformação, em pimentão cultivado em ambiente protegido nas estações sazonais verão/outono (V/O) e inverno/ primavera (I/P) (normal-plot for number of fruits (A) and fresh biomass of fruits (B) before and after the transformation, in green pepper cultivated in protected environments during summer-fall (V/O) or winter-spring (I/P) seasons). Santa Maria, UFSM. 2009. de frutos de abobrinha italiana cultivada em diferentes sistemas de irrigação. Revista Ceres 55: 590-595.

CONAGIN A; NAGAI V; IGUE T. 1993. Efeito da falta de normalidade em testes de homogeneidade das variâncias. Bragantia 57: 203-214.

COUTO MRM; LÚCIOAD; LOPES SJ; CARPES RH. 2009. Transformação de dados em experimentos com abobrinha italiana em ambiente protegido. Ciência Rural 39: 17011707.

CUNHA AR; ESCOBEDO JF. 2003. Alterações micrometeorológicas causadas pela estufa plástica e seus efeitos no crescimento e produção da cultura do pimentão. Revista Brasileira de Agrometeorologia 11: 15-26.

CUNHAAR; ESCOBEDO JF; KLOSOWSKI ES. 2002. Estimativa do fluxo de calor latente pelo balanço de energia em cultivo protegido de pimentão. Pesquisa Agropecuária Brasileira 37: 735-743.

DRAPER NR; SMITH H. 1981. Applied regression analysis. New York: John Wiley, 709p.

FILGUEIRA FAR. 2000. Novo manual de Olericultura: tecnologia moderna na produção e comercialização de hortaliças. Viçosa: UFV, 402p.

GRIFFITHS DA. 1980. Interval estimation for the three-parameter lognormal distribution via the likelihood function. Applied Statistics 29: $58-68$

HILL BM. 1963. The three-parameter lognormal distribuition and Bayesian analysis of a pointsource epidemic. Journal of the American Statistical Association 58: 72-84.

LOPES SJ; STORCK L; HELDWEIN AB; FEIJO S; ROS CA. 1998. Técnicas experimentais para tomateiro tipo salada sob estufas plásticas. Ciência Rural 28: 193-197.

LORENTZ LH; LÚCIO AD; BOLIGON AA; LOPES SJ; STORCK L. 2005. Variabilidade da produção de frutos de pimentão em estufa plástica. Ciência Rural 35: 316-323.

LÚCIO AD; SOUZA MF; HELDWEIN AB; LIEBERKNECHT D; CARPES RH; CAVALHO MP. 2003. Tamanho da amostra e método de amostragem para avaliação de características do pimentão em estufa plástica. Horticultura Brasileira 21: 181-185.

LÚCIO AD; CARPES RH; STORCK L; LOPES SJ; LORENTZ LH; PALUDO AL. 2008. Variância e média da massa de frutos de abobrinha-italiana em múltiplas colheitas. Horticultura Brasileira 26: 333-339.

MELLORM;LÚCIOAD; STORCKL;LORENTZ LH; CARPES RH; BOLIGON AA. 2004. Size and form of plots for the culture of the italian pumpkin in plastic greenhouse. Scientia Agricola 61: 457-461.

SOUZA MF; LÚCIO AD; STORCK L; CARPES RH; SANTOS PM; SIQUEIRA LFF. 2002. Tamanho da amostra para peso da massa de frutos, na cultura da abóbora italiana em estufa plástica. Revista Brasileira de Agrociência 8: 123-128.

STORCK L; LOPES SJ; ESTEFANEL V; GARCIA DC. 2000. Experimentação vegetal. 1ed. Santa Maria: UFSM. 198p.

YAMAMURA K. 1999. Transformation using $(\mathrm{x}+0.5)$ to stabilize the variance of populations. Journal Researches on Population Ecology 42: 229-234 